\title{
31 AUTOMATING PARTNER SELECTION FOR A VIRTUAL ORGANIZATION
}

\section{Tomasz Janowski", Liu Yonghe* and Huang Biqing* The United Nations University, Macau}

\begin{abstract}
Establishing a virtual organization requires not only being able to identify individual member enterprises but also prescribe the roles they should play in the organization. This becomes a difficult task when selection is not only based on static competencies but also on how the enterprise is able to dynamically deliver services to its clients, within the area of its competence. In this paper we present a simple model which explains the behavior of a virtual organization in terms of the services it can offer to and receive from its environment, by means of the services by its members and service-based interactions between them. We also describe how the model could be used to define and automate the problem of partner selection: produce an architecture (members and their roles) to deliver the required service.
\end{abstract}

\section{INTRODUCTION}

Establishing a virtual organization requires the basic infrastructure for communication and information sharing between individual enterprises to be already in place, not unlike for other business applications or distributed computing at large. While this level of support is necessary it is also insufficient; the added value comes from the higher-level functions able to address specific problems facing a virtual organization. One of such problems is partner selection.

Instead of relying only on the known companies we would like to actively seek information about potential partners on the Internet, taking into account not only the static "competence" of an enterprise but how it is able to dynamically deliver services to its clients, within the area of its competence. The following problems exist when we use for this purpose existing Internet search tools: (1) it is not clear which keywords we should use to describe what we look for, (2) the search is likely to produce an abundance of largely irrelevant and out-of-date data, (3) it is unclear

\footnotetext{
- The United Nations University, International Institute for Software Technology, P.O. Box 3058, Macau, e-mail: tj@iist.unu.edu

* From the CIMS Research and Engineering Center at Tsinghua University, Beijing, China
} 
which criteria we should apply to such data and (4) the data gives little indication as to the roles different partners could play in the organization. Relying on the generalpurpose support, here an Internet search engine means we also have to rely on human assistance to interpret the low-level results. In this paper we aim to study partner selection as an infrastructure support for a virtual organization, reducing the role of human users to decision-making, given possible partners and the roles they could play in the organization, instead of interpreting raw data.

In order for this support to be possible we have to state very clearly what is the goal of the organization, the roles of its members contributing towards this goal and possible interaction between them. Not only description of the goals should be machine-readable but carry enough semantic information to allow software to make decisions without asking for human assistance, to compare what the organization needs and what the potential member has to offer. We adopt a model that explains the behavior of a virtual organization in terms of the services it can offer to and receive from its environment, by means of the services by individual members and service-based interactions between them. The concept of a service includes a range of possible interactions between the entities in the marketplace - suppliers, manufacturers, retailers, logistics providers, and stockholders - all potential members of the organization. For example: delivery of a certain number of subproducts from the supplier to the manufacturer (supplier is a service provider, manufacturer is a service consumer), transport of products between the manufacturer and the retailer (provided by a transportation company), marketing of a product for the customers living in a certain area (provided by a marketing company) etc. We can describe the services atomically, built them from some other services and compare services with an ordering which decides if one service is at least as "good" as another one. We treat all entities in the marketplace as both providers and consumers of services, often at the same time: a service describes what the entity requires from its environment and what it can deliver when it receives what it needs. This conditional delivery of services provides an external view on individual entities, which is all we need to know for partner selection.

A virtual organization delivers its services using the services offered by its members and service-based interactions between them. Partner selection starts with a service the organization should deliver to its customers. If we can find an entity in the marketplace which can solely deliver this service then the search is finished. In general, however, the entity will only deliver the service if provided with some other services. Then we continue looking for the entities which are able to deliver those auxiliary services, and so on. With each iteration we build the links between the entities in the marketplace (supply-chains using the product analogy), deciding on the members of the virtual organization and the roles they should play (services). The search is finished when the organization is self-contained and does not require any more services from its environment, or on purpose decides to rely on the environment to deliver some of them. Such external services can be determined at the start or during the process of selection. The result is a possible architecture (members and their roles) of the virtual organization to deliver the required service.

The rest of the paper is organized as follows. Section 2 presents the servicebased model for a virtual organization. Section 3 illustrates the model by a simple example. Section 4 applies the model to formulate the problem of partner selection. Section 5 provides discussion, comparisons and directions for our future work. 


\section{SERVICE-BASED MODEL}

We introduce in this section a service-based model for a virtual organization. The aim is to formulate the problem of partner selection. The model is given in a formal notation of RAISE: Rigorous Approach to Industrial Software Engineering (RAISE, 1992) (RAISE, 1995). In the sequel we introduce different elements of the model: services, service-providers, service-based interactions and service composition.

\section{Service}

A service represents what the entity in the marketplace requires for carrying out its business activities and what such activities can deliver. At this stage we are not interested in the details of a service, therefore we introduce an abstract type Service which values represent possible services. In RSL, a type represents a collection of values together with some operations on them.

type

Service

In addition to describing services we would like to be able to match them: one service represents what is needed by an entity in the marketplace and another service represents what some other entity can provide. How to decide if those two services match? This may differ between cases, but in general we should be able to provide an ordering between services which represents this matching: if $s \leq t$ then $t$ is matching the service required by $s$. We require that the service ordering is reflexive (any service satisfies itself), anti-symmetric (it two services satisfy each other then they are equal) and transitive (if one service satisfies the second and the second satisfies the third then also the first satisfies the third). We define the ordering as a Boolean function $\leq$ on the pairs of services, as below.

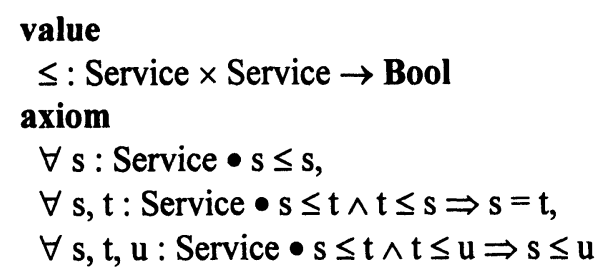

We also assume two special values of the type Service representing the upper and the lower bounds of the service ordering: top and bottom. bottom is the least demanding service which can be satisfied by any service whatsoever, top is the most demanding service that is only satisfied by itself.

value

top : Service $\bullet(\forall s$ : Service $\bullet s \leq$ top $)$,

bottom : Service $\bullet(\forall \mathrm{s}$ : Service $\bullet$ bottom $\leq \mathrm{s})$ 


\section{Service Provider}

We treat each entity in the marketplace as a possible service provider. We are not interested in the details of how the delivery of a service takes place, so we define a provider as a value of an abstract type Provider.

\section{type}

\section{Provider}

Values of this type will represent service providers in a particular state, which state can change over time. In general, the entity can only provide a given service conditionally, given that it can receive some other services from the environment. Function provides represents static provision of services. It takes three arguments: the input service, the provider and the output service, and returns true or false depending if the provider is able to deliver an output service when given the input service. We constrain this function with respect to the ordering on services. One axiom says that any provider can deliver an output service given an input service which is at least as strong. In this situation no new service is produced, the entity delivers the service produced elsewhere without any change. Another axiom allows strengthening the input service and weakening the output service.

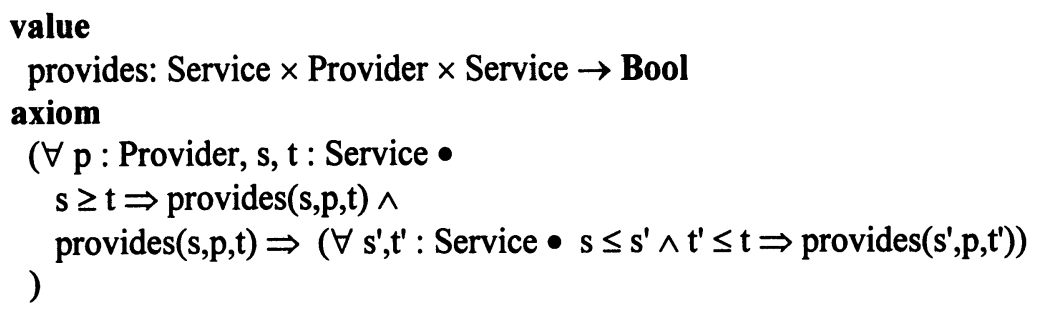

Some special cases of this function include: provides $(s, p, s)$, given $s$ as input $p$ can produce $s$ itself; provides (top, $p, s)$, given top as input $p$ can deliver any service $s$; provides (bottom, $p, s$ ), p can deliver $s$ on its own. Function provides takes into account only static provisions for services, for a given state of the provider. But this state can change when services are delivered. The function trans carries out this change of state. It takes the same arguments as function provides but returns the new provider. The function is partial in the sense that the result is only meaningful for those arguments for which provides returns true i.e. the delivery of a service is possible in a given state.

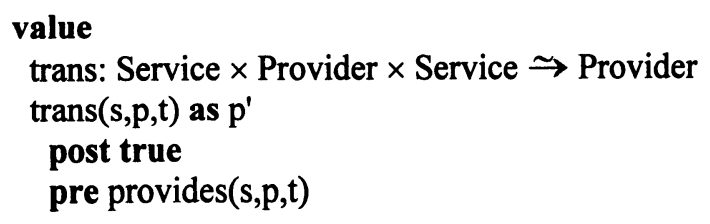




\section{Service-Based Interactions}

Given the definitions in the last section it is possible that the provider can itself deliver services which support some of its other services, becoming also a service consumer. This represents the level of being self-sufficient for the service provider, beyond which it certainly needs input from its environment. This maximum of the service delivery is represented by the function provides_star: if provides_star $(s, p, t)$ then either provides $(s, p, t)$ directly or there exists an intermediate service $s^{\prime}$ which can be output by $p$ after receiving $s$ and such that $t$ can be delivered in the new state trans $\left(s, p, s^{\prime}\right)$.

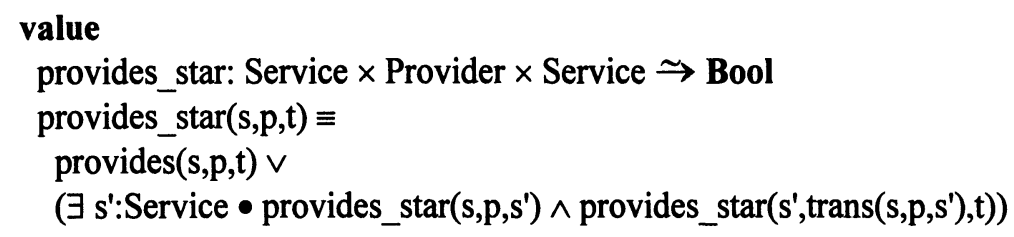

In general, a service produced by one provider will be consumed by another. Suppose we have a set of service providers and we would like to describe how they behave together, by delivering services individually and also interacting with each other. We first introduce the function virtual which given a set of service providers produces a single one.

\section{value \\ virtual: Provider-set $\rightarrow$ Provider}

Instead of defining this function explicitly, we define implicitly the behavior of the resulting service provider in terms of the functions provides and trans. The first axiom says that any service which can be delivered by a single provider can be also delivered by the virtual provider, i.e. if $\operatorname{provides}(s, p, t)$ then also provides $(s, \operatorname{virtual}(p s), t)$ given that $p \in$ ps. Only the state of this one provider will change, i.e. trans $(s, \operatorname{virtual}(p s), t)$ equals the result of virtual applied to ps where instead of $p$ we have trans $(s, p, t)$.

axiom

( $\forall$ ps:Provider-set, p,p':Provider, s,t:Service •

$\mathrm{p} \in \mathrm{ps} \wedge$ provides $(\mathrm{s}, \mathrm{p}, \mathrm{t}) \Rightarrow$

provides $(\mathrm{s}, \operatorname{virtual}(\mathrm{ps}), \mathrm{t}) \wedge$

)

$$
\operatorname{trans}(\mathrm{s}, \operatorname{virtual}(\mathrm{ps}), \mathrm{t})=\operatorname{virtual}(\mathrm{ps} \backslash\{\mathrm{p}\} \cup\{\operatorname{trans}(\mathrm{s}, \mathrm{p}, \mathrm{t})\})
$$

The second axiom includes the possibility for two providers to actually interact. If one can deliver a service which another can consume, then the interaction between them becomes possible. Their states will change simultaneously, taken nondeterministically for each one but not affecting the rest. 
axiom

( $\forall$ ps:Provider-set, p,q:Provider, s,t:Service •

( $\exists s^{\prime}:$ Service •

$\mathrm{p} \in \mathrm{ps} \wedge \mathrm{q} \in \mathrm{ps} \wedge \operatorname{provides}\left(\mathrm{s}, \mathrm{p}, \mathrm{s}^{\prime}\right) \wedge \operatorname{provides}\left(\mathrm{s}^{\prime}, \mathrm{q}, \mathrm{t}\right)$

$\Rightarrow \operatorname{provides}(\mathrm{s}, \operatorname{virtual}(\mathrm{ps}), \mathrm{t}) \wedge$ )

$\operatorname{trans}(\mathrm{s}, \operatorname{virtual}(\mathrm{ps}), \mathrm{t})=\operatorname{virtual}\left(\mathrm{ps} \backslash\{\mathrm{p}, \mathrm{q}\} \cup\left\{\operatorname{trans}\left(\mathrm{s}, \mathrm{p}, \mathrm{s}^{\prime}\right), \operatorname{trans}\left(\mathrm{s}^{\prime}, \mathrm{q}, \mathrm{t}\right)\right\}\right)$

)

We treat a virtual organization as a set of interacting service providers. Function virtual represents the abstracted behavior of the organization which on outside appears like a single provider. One obvious advantage is to minimize the number of modeling concepts. But more important is allowing for different levels of abstraction to describe the organization: on the concrete level the organization is a set of interacting service providers, on the abstract level it is an entity which can interact with its environment by delivering/consuming services. This in turn allows us to justify design of a virtual organization with respect to its abstract specification, using refinement. We say that provider $q$ refines provider $p$, refines $(p, q)$, iff provides $(s, p, t)$ for any $s$ and $t$ implies provides $\left(s^{\prime}, q, t^{\prime}\right)$ for an $s^{\prime}$ which is not stronger than $s$ and $t$ ' which is not weaker than $t$, i.e. $q$ can deliver at least all services that $p$ can while it requires no more from the environment. This property must be also preserved under state-changes, i.e. the new states again refine each other: refine $\left(\operatorname{trans}(s, p, t), \operatorname{trans}\left(s^{\prime}, q, t^{\prime}\right)\right)$.

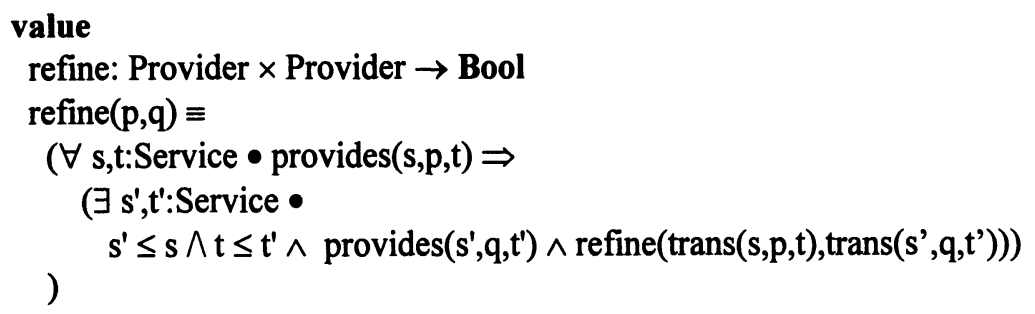

Given the required behavior described by the provider $p$, the set $p s$ of providers correctly implement this behavior iff $\operatorname{refine}(p, \operatorname{virtual}(p s))$.

\section{Composition of Services}

In this section we extend the model introduced so far by putting more structure on the type Service. We do so by service composition: concurrent, alternative and sequential, all defined as binary functions:

\section{value \\ con,alt,seq: Service $\times$ Service $\rightarrow$ Service}

Composition con $(s, t)$ describes that $s$ and $t$ are present simultaneously, alt $(s, t)$ that only one of $s$ or $t$ is present but we don't know which one, 
seq $(s, t)$ that service $t$ is delivered after $s$. We introduce some axioms which express requirements imposed by those interpretations, by equality over services and also using functions provides and trans.

Consider concurrent composition. The order in which we compose services concurrently is irrelevant, i.e. con is both commutative and associative. This justifies an extension of con into ext_con which takes a set of services instead of just two; the result for an empty set of services is bottom. Composing concurrently $s$ with bottom gives $s$. Also con gives a stronger service than those described by its components, i.e. $s \leq \operatorname{con}(s, t)$ and $t \leq \operatorname{con}(s, t)$.

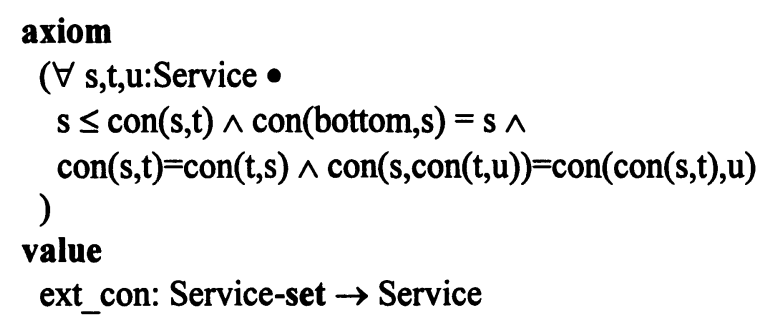

If $p$ can deliver concurrently $t 1$ and $t 2$ then it should also be able to deliver them individually, for the same input. But not the other way round: despite being able to deliver $t 1$ and $t 2$ individually, $\mathrm{p}$ may not be able to deliver them simultaneously. This becomes possible when we have separate entities that can simultaneously consume/deliver services, as in the virtual organization. In particular if provides $(s 1, p 1, t 1)$ and provides $(s 2, p 2, t 2)$ and if $p 1$ and $p 2$ are different then the composition of $\mathrm{p} 1$ and $\mathrm{p} 2$ can deliver concurrently con $(t 1, t 2)$ when provided concurrently with con $(s 1, s 2)$.

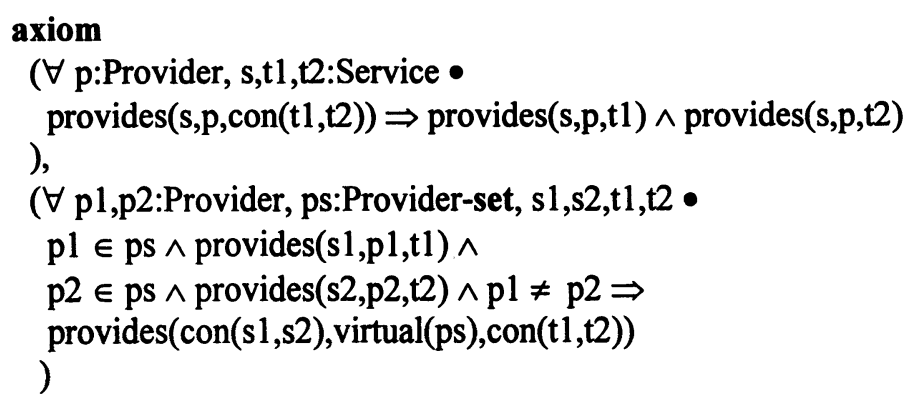

Concurrent services make also possible interactions between more than two providers: if $p$ can deliver $t$ given concurrently $\operatorname{con}(s 1, s 2)$, if $p 1$ and $p 2$ can deliver separately $s 1$ and $s 2$ given respectively $t 1$ and $t 2$, then their composition can deliver $t$ given concurrently con $(t 1, t 2)$.

Consider alternative composition. Like concurrency, the order in which we compose services is irrelevant, i.e. alt is both commutative and associative. Unlike concurrency, $s$ composed with itself gives $s$, i.e. alt is indepotent. Also concurrency gives a service which is equal or stronger than both of its components 
while alternative composition gives a service which is weaker (or equal); we are not sure which of the two services will be eventually chosen.

\section{axiom}

$(\forall \mathrm{s}, \mathrm{t}, \mathrm{u}:$ Service $\bullet$

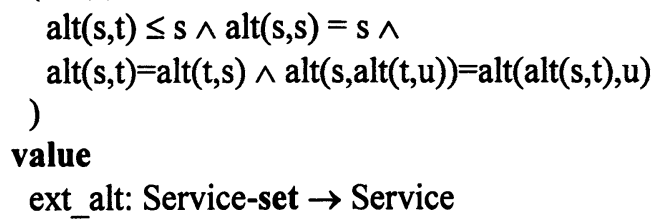

Because alt $(s 1, s 2)$ is equal or weaker than both $s 1$ and $s 2$, if $p$ can deliver $t$ given alternatively $s 1$ or $s 2$ then it can also deliver $t$ given only $s 1$ or only $s 2$. The opposite also holds: if $\mathrm{p} 1$ can deliver $t$ given $s 1$ and $\mathrm{p} 2$ can deliver $t$ given $s 2$ then $p 1$ and $p 2$ can deliver $t$ given alternatively $s 1$ or $s 2$.

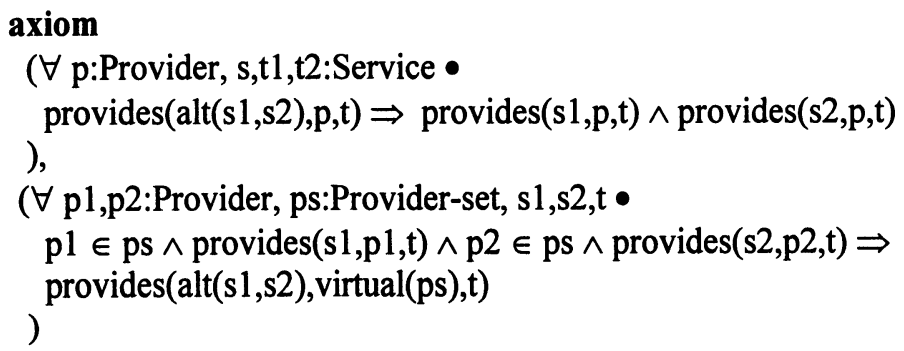

Consider sequential composition. Service $\operatorname{seq}(s, t)$ is stronger than $s$ itself $(s \leq s e q(s, t))$ and composition is associative but we cannot change the order in which $s$ and $t$ are delivered. Sequential delivery is inherently possible for every service provider, normally defined jointly by the functions provides and trans. Here we can also make it explicitly part of the service definition: if $p$ can deliver $t 1$ given $s 1$ and in the new state deliver $t 2$ given $s 2$ then it can also deliver sequentially $\operatorname{seq}(t 1, t 2)$ given sequentially $\operatorname{seq}(s 1, s 2)$.

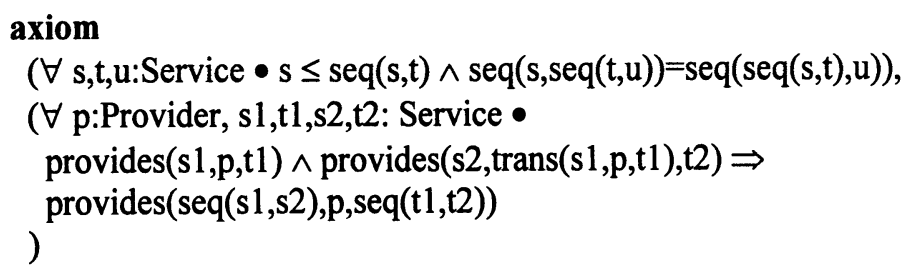

Like concurrency, sequential composition makes possible the interactions between more than two service providers. In particular, different services that are part of sequential composition can be satisfied (consumed) independently by separate providers. This way the links between service providers extend over time, involving entities that are able to change their states and change their behavior accordingly. 


\section{MANUFACTURING EXAMPLE}

Suppose a service means delivery of a certain quantity of a given product. We introduce the abstract type Product which values represent different products and a function bill from Product to maps from Product to Nat to represent how we can obtain products from sub-products. This map represents all sub-products of a given product and their quantities: how many items we need to assemble a single item of the final product. We constrain function bill by requiring that the quantity of each sub-product is non-zero and no product is a sub-product of itself.

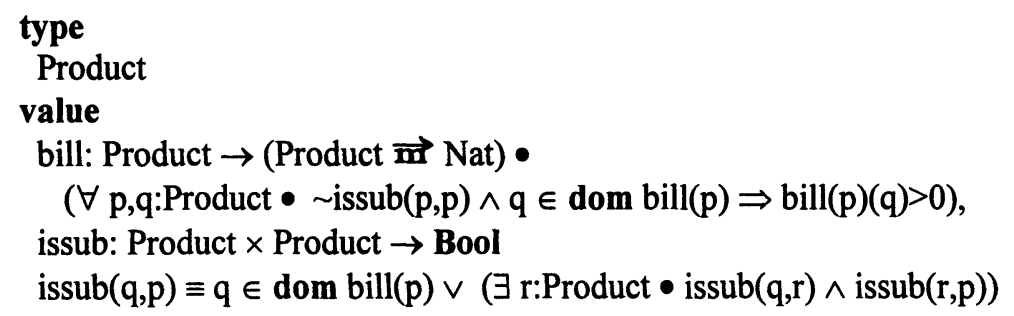

Let Nat 1 represent positive natural numbers. We define the type Service to include two kinds of values: bottom (no product is delivered) and $f$ in $(p, n)(n$ $\geq 1$ items of product $p$ are delivered). By underscore we mean that Service can also contain other values, to be defined later. The ordering $s \leq t$ is always true if $s=$ bott $\mathrm{m}$. If $s=f$ in $(p, n)$ then $s \leq t$ provided $t=f$ in $(p, m)$ for the same $p$ and $m \geq n$. It is easy to see that this definition satisfies the axioms of the partial order.

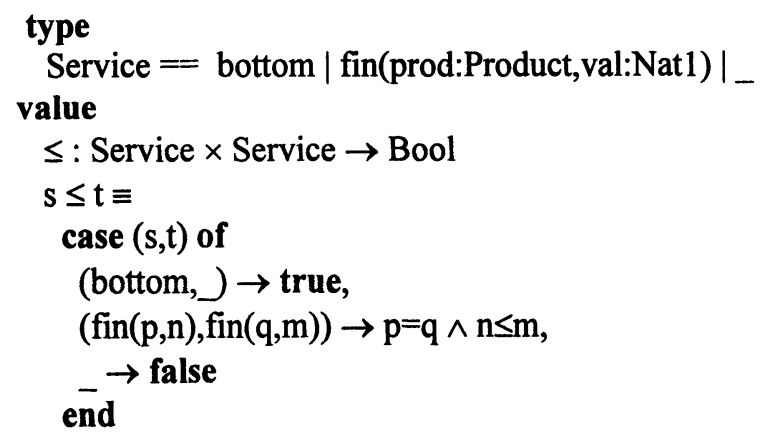

Suppose a service provider contains the stock for various products and is able to manufacture products from sub-products. We introduce two functions on the type Provider: shop returns the set of products that can be manufactured on the shopfloor and stock returns the number of items of a given product on stock.

value

shop: Provider $\rightarrow$ Product-set

stock: Provider $\times$ Product $\rightarrow$ Nat 
We also consider some operations on this model: store increments the stock for a given product, deliver decrements the stock and manufacture increments the stock for the product and decrements the stocks from all its sub-products, according to the bill. All take two arguments (service and provider) and accordingly change the state of the provider. Function manufacture takes two preconditions: the product can be manufactured and the stock contains all required sub-products.

\section{value}

store, deliver, manufacture : Service $\times$ Provider $\leadsto$ Provider

Function provides takes the input service, provider and the output service and decides if the service can be delivered: either the output is bottom or the stock is at least equal the quantity required by the output service or we can manufacture the missing part from existing sub-products. Function trans carries out the corresponding state-change: stores the result of the input service and delivers the output service (which may involve manufacturing the missing part of products).

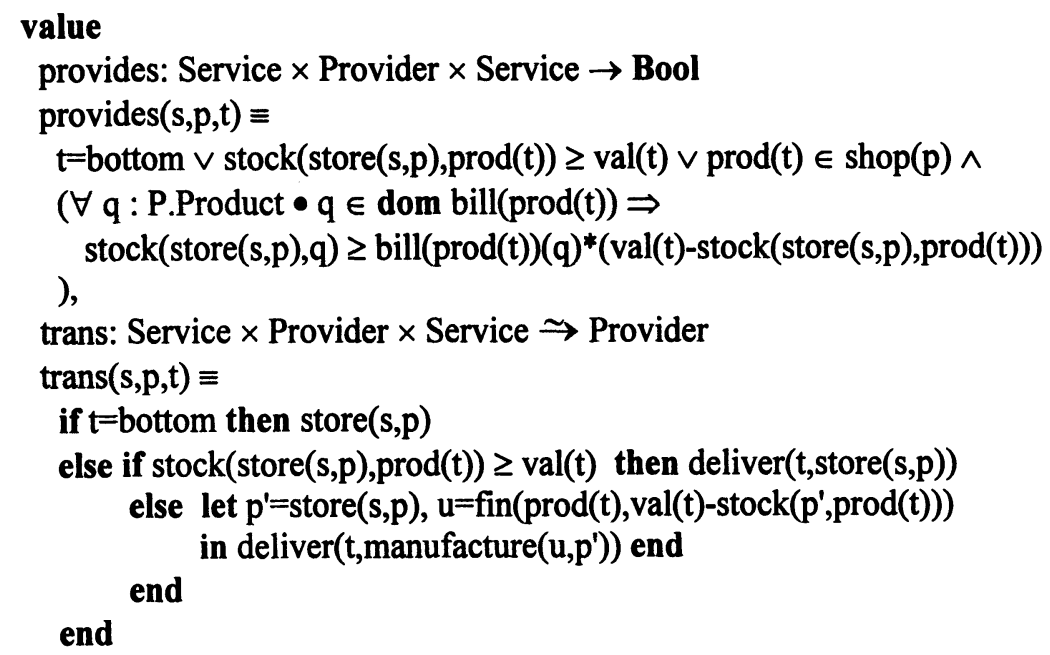

Based on those functions we define function virtual which produces the behavior of the virtual organization by summing up the sets of products which can be manufactured on the shopfloor and the stocks for all products. The resulting function indeed satisfies the axioms expressing what services (product and quantity) can be delivered by the virtual organization and how the state will change as a result.

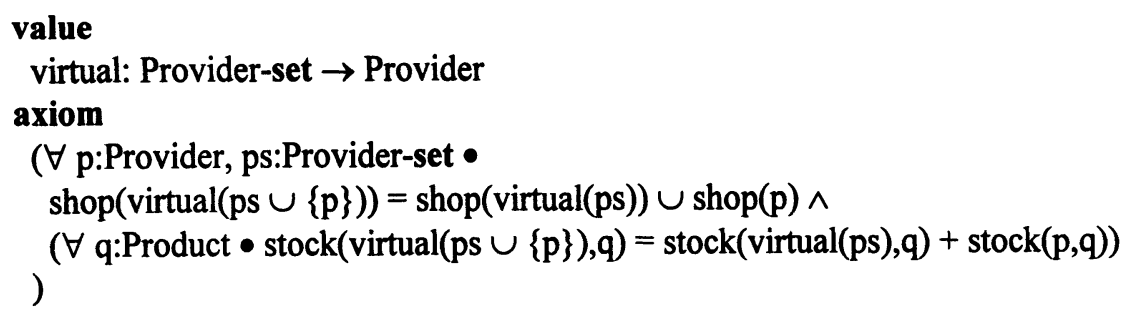




\section{AUTOMATING PARTNER SELECTION}

Based on the model in Section 2 we can formulate the problem of partner selection. The problem takes three inputs: the service we want to deliver, the set of providers we can choose from and the service we wish those providers can rely upon; this service is assumed to be provided by the environment. Both services may in fact represent sets of services, put together by means of concurrent or other composition. The output determines which of those providers can deliver the required service while relying on the assumed service. In addition to selecting service providers we wish to determine the roles they should play in the delivery of the output service.

The simplest case is a single provider in the set able to solely deliver the required service when given the input service. Function can_provide_1 decides if such a provider exists. Function provider_1 returns any such provider. In this simple case the organization consists of only a single entity, which role is simply to deliver the output service when given the input service.

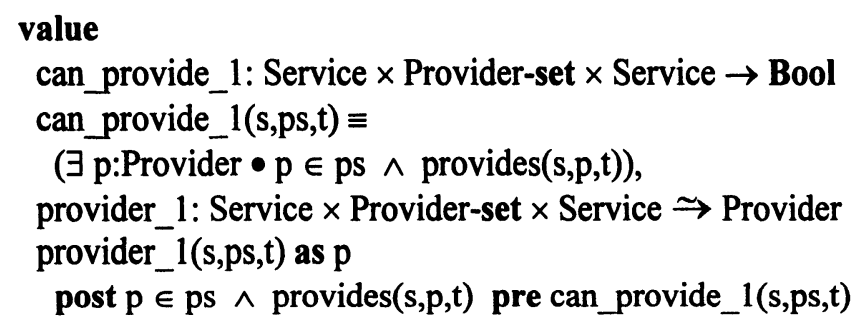

However, selection of a single entity that can deliver the required service on its own is not always possible. More often the combination of two or more entities can do the job which a single entity cannot. Below we consider the case of two providers. Function can_provide_2 decides if we can find two providers in the set that together can deliver the required service. Function provider_2 returns those two providers and a service which "links" them together. The first provider delivers this service when given the input service, the second delivers the output service while consuming the intermediate service. Their roles are set very precisely.

value

can_provide_2: Service $\times$ Provider-set $\times$ Service $\rightarrow$ Bool

can_provide_2 $2(s, p s, t) \equiv$

( $\exists$ p1,p2:Provider, u:Service •

$\{\mathrm{p} 1, \mathrm{p} 2\} \subseteq \mathrm{ps} \wedge \operatorname{provides}(\mathrm{s}, \mathrm{p} 1, \mathrm{u}) \wedge \operatorname{provides}(\mathrm{u}, \mathrm{p} 2, \mathrm{t}))$, Provider

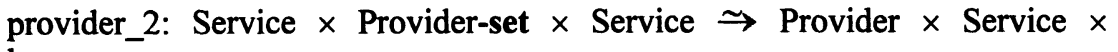

provider_2(s,ps,t) as (p1,u,p2)

post $\{\bar{p} 1, \mathrm{p} 2\} \subseteq \mathrm{ps} \wedge$ provides $(\mathrm{s}, \mathrm{p} 1, \mathrm{u}) \wedge \operatorname{provides}(\mathrm{u}, \mathrm{p} 2, \mathrm{t})$

pre can_provide_2(s,ps,t) 
The resulting organization consists of exactly two entities. One step further is to consider a chain of providers such that the first provider consumes the input service, the last delivers the output service, and from the second onwards, every provider consumes the service the previous one delivers. Function can_provide_chain decides if such a chain exists and provider_chain returns the list of providers with corresponding output services. For instance $\langle(p 1, u),(p 2, t)\rangle$ is a twomember chain where $p 1$ delivers $u$ given $s$ and $p 2$ delivers $t$ given $u$. We do not exclude that $\mathrm{p} 1$ and $\mathrm{p} 2$ denote the same provider, but this requires to take into account state-changes. The last service is always the final required service.

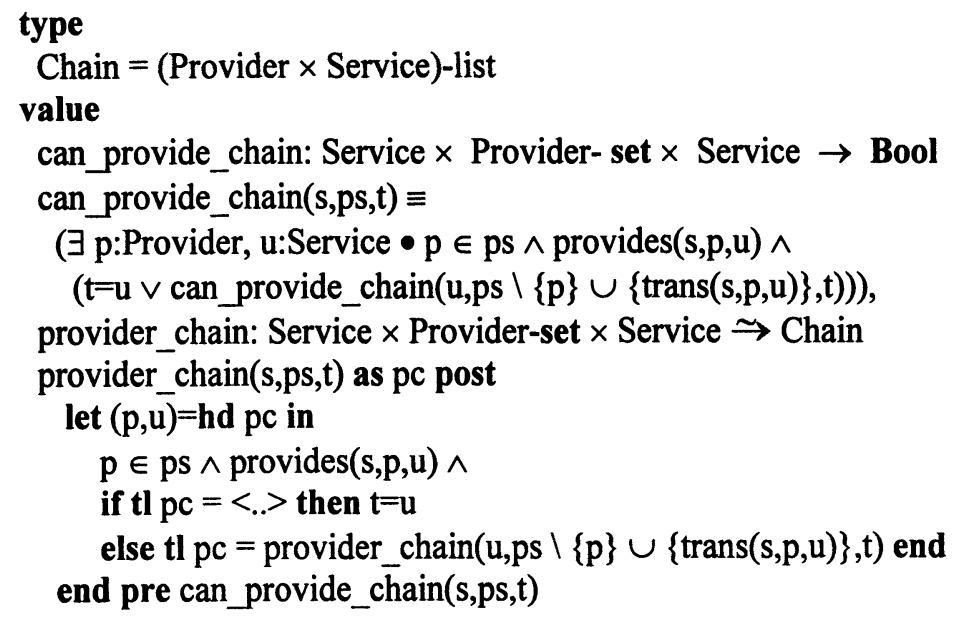

The resulting virtual organization consists in general of several entities that are linked sequentially. More complex configurations are also possible, say in the form of a tree which decides that a given provider receives input partly from the environment (if weaker than the given service) and partly from the providers in the set, possibly several of them. Each one can in turn rely on the inputs received partly from the environment and partly from other, one or more, providers. We can decide in the tree structure if the input service combines component services concurrently, sequentially or alternatively, with corresponding constraints on a single provider occurring in more than one place in the tree. For instance, if the provider occurs several times in a single branch then we should take into account the fact that its state will change, from the leaf towards the root. But the tree structure is not very appropriate if we are to allow the same provider to occur concurrently in several sub-trees, having to capture an arbitrary interleaving of its state-changes. A kind of graph structure, similar to transition systems, would have to be used instead. We plan to investigate such issues in a companion paper.

More generally, we may like to derive some rules to follow for carrying out partner selection. Suppose $t$ is the output service, $s$ the input service and $p s$ is the set of providers we can choose from. We could make the whole selection task easier if we reduce this problem into the one where we receive stronger input, say $s \leq s^{\prime}$, and are required to produce weaker output, say $t ' \leq t$. This is described by the 
functions stronger_input and weaker_output below. We could also combine those functions together.

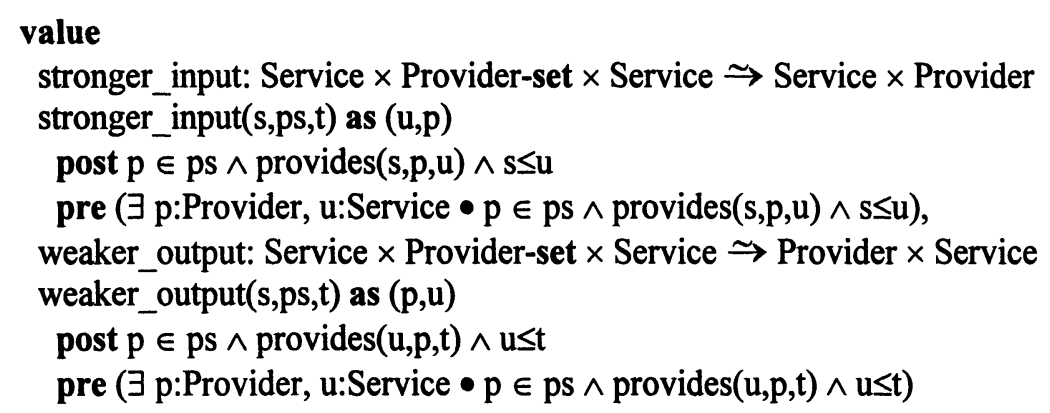

Another methods of reduction is to look at the structure of the input/output service to see if we can decompose the problem into a number of simpler subproblems. In particular, if the input service is built with alternative composition, say $s=a l t(s 1, s 2)$, then the problem for $s$ and $t$ reduces into two problems: for $s 1$ and $t$, and $s 2$ and $t$. Solving one of those problems is enough to solve the original problem because alt $(s 1, s 2) \leq s 2$ as well as alt $(s 1, s 2) \leq s 2$, i.e. both assumptions are stronger than the original assumption.

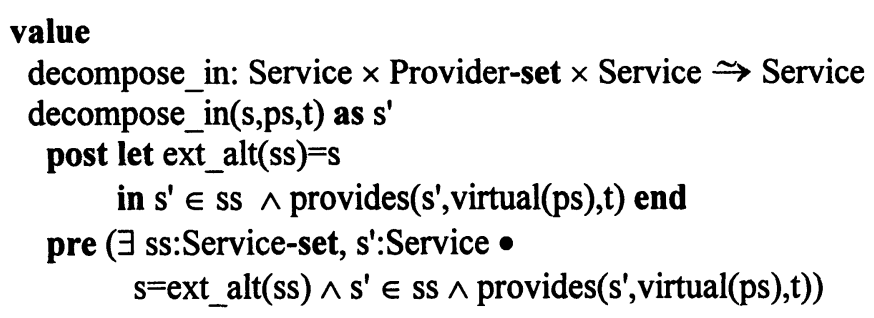

Even more important is to be able to decompose output which is given with concurrent composition, say $t=\operatorname{con}(t 1, t 2)$. Then the problem for $s$ and $t$ reduces into two problems: for $s$ and $t 1$, and $s$ and $t 2$. Unlike before, we have to solve both problems, not just one of them. Moreover, we must do so with disjoint sets of providers. This is described by the function decompose_out.

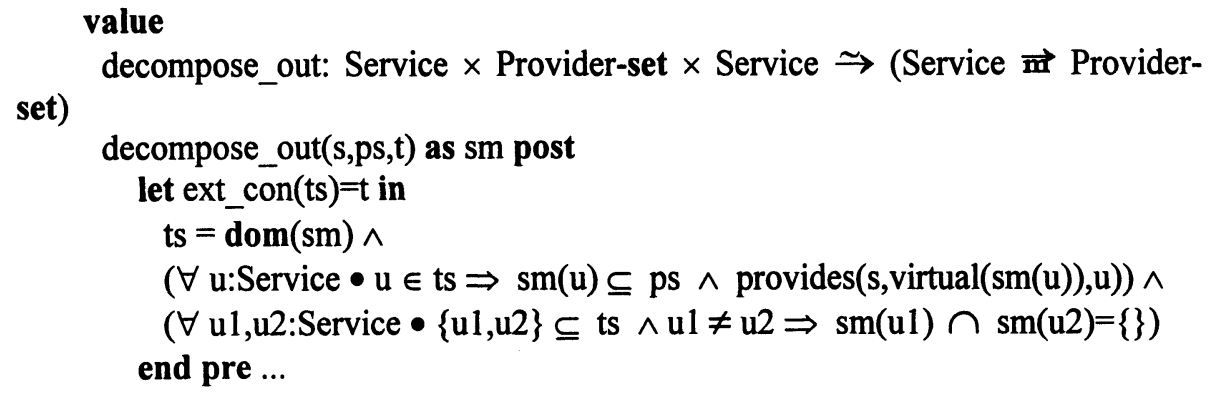




\section{CONCLUSIONS}

In this paper we considered the problem of partner selection for a virtual organization, in particular how the problem could be automated. We decided not to place any particular assumptions about the kind of business the organization is involved with, be it manufacturing, logistics, marketing etc. We also decided that competence is on its own insufficient for selecting a partner, but this selection should be rather based on the dynamic provision for services the partner can offer to its environment. Finally, we decided that before we can come with any implementation for this problem, we should be able to first define and formalize it. Underlying such decisions is what we consider to be crucial for a systematic treatment of the issues surrounding the concept of a virtual organization: abstraction. We would like to be able to tackle such issues in the general setting, then only instantiate them to concrete situations, rather than consider them for specific kinds of businesses or even particular enterprises. We believe this is part of the general question to what extent we can treat a virtual organization (with no added adjectives) as the topic of analytical, not only empirical or technological, study.

The need for abstraction is particularly acute for formulating and solving the problem of partner selection, where different partners may be involved in various kinds of business activities and offer each other a variety of services. Heterogeneity in this case is part of life. In order to overcome such differences as well as factor out necessary similarities, we presented a model that represents business activities as services and business entities as service providers. A service received a formal definition with an ordering to allow matching services: one service is provided, another one is required. A service provider is given a formal operational semantics describing its changing ability to conditionally deliver services to its environment (which includes customers as well as other service providers). The state of the provider can change over time, i.e. with every delivery of a service. Not unlike its members, a virtual organization is treated as a service provider who delivers its services by means of the services by its members and service-based interactions between them. With this simple conceptual model we were able to formalize the problem of partner selection for a virtual organization starting from the services the organization is required to provide to its environment (its business goal) and the services it is allowed to rely upon (its assumptions). Selection was carried out by searching for those providers who are able to fulfill the goal under given assumptions. When formulating such goals and assumptions we could use service composition: concurrent (services provided simultaneously), sequential (provide one service, then another one) or alternative (one of two services, chosen nondeterministically). The model provides not only the possibility to actively seek partners through advanced technology, but also the mechanism to dynamically organize the structure within the virtual organization. It describes the behavior of individual members and their interactions. Unlike a traditional organizational view of an enterprise that concentrates on the internal operations, we took a crossenterprise view of how enterprises can effectively cooperate at an abstract level.

The concept of a virtual organization (Davidow and Malone, 1992) (Browne, 1995) (Camarinha-Matos and Afsarmanesh, 1997) proved to be of the lasting 
interest, evolving from the early ideas of Computer Integrated Manufacturing (Teicholz and Orr, 1987) and Enterprise Integration (Vernadat, 1996), with several representative approaches to enterprise modeling like e.g. GRAI (Zanettin et al, 1989), CIMOSA (AMICE, 1993), PERA (Williams, 1994) or GERAM (GERAM, 1998). We have seen in recent years several conferences dedicated, wholly or partly to virtual organizations, e.g. (Afsarmanesh et al, 1998) (Schonsleben and Buchel, 1998) (Bernus and Nemes, 1996). We have also seen several projects, notably PRODNET (Garita et al, 1997) (Production Planning and Management in an Extended Enterprise) and NIIIP (National Industry Information Infrastructure Protocol). We believe the work presented here complements existing results about virtual organizations. Most published results assume that the virtual organization already exists, while we concentrate on its creation. Often the main criterion considered for choosing a partner is its competence, a rather static attribute. We consider instead how the partner is able to dynamically deliver services to its clients, which is more adequate for the connected, on-line business. Most of the published results concentrate on the enabling technologies, paying less attention to the organizational and management issues. We believe such issues are critical for the success of the virtual organization. We also understand technology today is changing very rapidly, but the concept (virtual organization) and the problems related to it will remain. They are simply of the lasting interest and value. We seek to understand those problems independently from the technology used today, which is one of the reasons we chose to present the model for a virtual organization formally; the need for a more formal treatment of enterprise models have been observed before (Nemes et al, 1996) (Gruninger and Fox, 1996). Another reason is the need for abstraction. This work extends the model for a virtual manufacturing organization in (Janowski et al, 1998a) and its semantics in (Janowski et al, 1998b).

This paper presents work in progress. There are several directions we plan to continue our study. We plan to further develop the model of services, with new methods of composition (e.g. recursive definition of a service which repeats over time), quantitative comparison of services (based on the levels of attributes like price, reliability, servicing etc.), probabilities assigned to the services composed alternatively (one delivered with probability a, another one with probability 1-a) etc. We plan to further develop the model of service providers with various methods to compose them together (so far we only considered providers as members of a set), for example: $(s / t) \cdot p$ denotes a provider which first delivers service $s$ given $t$ and then behaves like the provider $p ; p+q$ behaves like either $p$ or $q$ depending on which service assumptions are satisfied first; $p>q$ two providers acting concurrently but also interacting by $p$ providing services to $q ; p<q$ two providers acting concurrently but also interacting by $p$ receiving services from $q ; p \backslash s$ behaves like $p$ but not delivers any $s^{\prime}$ which is stronger of equal $s ; p / t$ behaves like $p$ but not accepts any $t$ ' weaker than or equal $t$; etc. Similar like in process algebras (Hoare, 1985) (Milner, 1989), this should provide for an expressive architecture-description language for a virtual organization. We plan to extend semantics of service providers to allow for non-deterministic choice of the future state by means of function trans which returns the set of providers, not a single one. We carried out in this paper an example from manufacturing to illustrate the model, where a service denotes delivery of a given quantity of products and a service provider is a model of 
a manufacturing enterprise. We plan to build more examples with different service models, say representing logistics (a service is a pair of locations in the communication graph), marketing (extending (Janowski et al, 1998c)) but also more detailed manufacturing models which take into account delivery time, price etc. Finally, we plan to build prototype applications for demonstration of the servicebased model for a virtual organization on the Internet.

\section{REFERENCES}

1. Afsarmanesh $\mathrm{H}$, Camarinha-Matos $\mathrm{L}$ and Marik V, editors. Proceedings of the 3rd IEEE/IFIP Conference on Information Technology for Balanced Automation Systems in Manufacturing. Chapman and Hall, 1998.

2. AMICE. CIMOSA: Open System Architecture for CIM. Springer Verlag, 1993.

3. Bernus $P$ and Nemes L, editors. Modelling and Methodologies for Enterprise Integration. IFIP, Chapman and Hall, 1996.

4. Browne J. The Extended Enterprise: Manufacturing and The Value Chain. In Camarinha-Matos L and Afsarmanesh H, editors, BASYS95. Chapman and Hall, 1995.

5. Camarinha-Matos L. and Afsarmanesh H. Virtual Enterprise: Life Cycle Supporting Tools and Technologies. In Handbook of Life Cycle Engineering: Concepts, Tools and Techniques, Chapman and Hall, 1997.

6. Davidow W.H. and Malone M.S. The Virtual Corporation: Structuring and Revitalizing the Corporation for the 21st Century. Harper Collins, New York, 1992.

7. Garita C, Camarinha-Matos L, Afsarmanesh $\mathrm{H}$ and Lima C. Towards an Architecture for Virtual Enterprise. In Proceedings of the 2nd World Congress on Intelligent Manufacturing Processes and Systems, 1997.

8. Gruninger M. and Fox M.S. The Logic of Enterprise Modelling. In Modelling and Methodologies for Enterprise Integration, IFIP, 1996.

9. Hoare C.A.R. Communicating Sequential Processes. Prentice Hall International, 1985.

10. IFIP-IFAC Task Force. GERAM: Generalised Enterprise Reference Architecture and Methodology. June 1998.

11. Janowski T, Lugo GG and Zheng H. Composing Enterprise Models: The Extended and The Virtual Enterprise. In 3rd IFIP/IEEE Conference on Information Technology for Balanced Automation Systems in Manufacturing, Chapman and Hall, 1998.

12. Janowski $\mathrm{T}$, Zheng $\mathrm{H}$ and Lugo GG. Market-Driven Symbolic Execution of Models of Manufacturing Enterprises. In 2nd IEEE International Conference on Formal Engineering Methods, IEEE Computer Press, 1998.

13. Janowski T, Atienza RV and Lugo GG. Integrating Enterprise Models and Models for Marketing Analysis. In 2nd IFIP Conference on Design of Information Infrastructure Systems for Manufacturing, Chapman and Hall, 1998.

14. Milner R. Communication and Concurrency. Prentice Hall, 1989.

15. Nemes L, Bernus P and Morris R. The Meaning of an Enterprise Model. In Modelling and Methodologies for Enterprise Integration, 1996.

16. The RAISE Language Group. The RAISE Specification Language. BCS Practitioner Series. Prentice Hall, 1992.

17. The RAISE Method Group. The RAISE Development Method. BCS Practitioner Series. Prentice Hall, 1995.

18. Schonsleben $P$ and Buchel $A$, editors. Proceedings of the IFIP International Working Conference on Organizing the Extended Enterprise. Chapman and Hall, 1998.

19. Teicholz E and Orr J. Computer Integrated Manufacturing Handbook. McGraw-Gill, 1987.

20. Vernadat F. Enterprise Modelling and Integration. Chapman and Hall, 1996.

21. Williams T. The Purdue Enterprise Reference Architecture. Computers in Industry, 24(2), 1994.

22. Zanettin M, Roboam M and Pun L. GRAI: Integrated Methodology to Analyse and Design Manufacturing Systems. Computer Integrated Manufacturing Systems, 2(2):82--98, 1989. 